\title{
Outage Probability Analysis of Cooperative Communications over Asymmetric Fading Channel
}

\author{
Sudhan Majhi, Youssef Nasser and Jean François Hélard \\ National Institute of Applied Sciences of Rennes
}

France

\section{Introduction}

Cooperative relaying is a promising technology for future wireless communications. It is mostly applicable to the small dimensional and limited power devices, which cannot use the conventional multiple input multiple output (MIMO) technology to obtain the advantages of MIMO. It can benefit most of the leverages of MIMO such as array gain, diversity gain, spatial multiplexing gain and interference reduction without using the conventional MIMO technology Liu et al. (2009); Laneman et al. (2004); Paulraj et al. (2004). Since the original signal is forwarded by relay nodes, the performance of the relaying network depends on the relaying process of the relay nodes and fading characteristic of their links. Classically, relay network has three links source-destination (S-D), source-relay (S-R) and relay-destination (R-D) and the relaying processes are classified as amplify-and-forward (AF), decode-andforward (DF) and compress-and-forward (CF) Krikidis \& Thompson (2008); Nosratinia et al. (2004).

The diversity of the relaying network depends on the degree of freedom of the network. For repetition-based relaying, the degree of freedom increases with the number of relay nodes when the system is an half duplex and use a time division duplex Zhao et al. $(2005,2007)$. However, it suffers spectral efficiency with increase in the number of relay nodes in the network. On the other hand, opportunistic relaying uses only one relay node to forward source data to the destination and its degree of freedom is two. It has higher spectral efficiency and better outage performance than that of repetition-based relaying.

The performance of the relaying network depends on the fading characteristic of S-D, S-R and R-D links, i.e. diversity of the relaying networks depends on the location of the relay nodes and its surrounding environment. In practice, cooperative nodes are usually located in different geographical locations and at different distances with respect to $\mathrm{S}$ and $\mathrm{D}$. The signal in one link may be in line of sight (LOS) situation and other links may be in NLOS situation. For example, fixed relay nodes are used for forwarding source's data to a specific region (e.g. tunnel, behind of the building) and they often use directional antennas, so the R$\mathrm{D}$ link is likely to be a LOS situation. However, we cannot assume such scenario for other links specially when $\mathrm{D}$ is in a shadowing region with respect to S. In other words, one link may undergo Rician fading channel and others links may undergo Rayleigh fading channel. Such scenario is refereed to as asymmetric fading channel. This channel scenario can also be 
seen in cooperative cognitive radio where secondary terminal works as a relay. In addition, all the links, i.e., $\mathrm{S}$ to $\mathrm{i}^{\text {th }}$ relay $\left(\mathrm{S}-\mathrm{R}_{i}\right)$ and $\mathrm{i}^{\text {th }}$ relay to $\mathrm{D}\left(\mathrm{R}_{i}-\mathrm{D}\right)$, may be independent but nonidentically distributed (i.n.d) fading channels. Therefore, a complete outage performance study of cooperative relaying for such asymmetric and i.n.d fading channels is required.

The outage probability of relaying networks over symmetric fading channel, in which all the links undergo the same fading distribution, is provided in several works Hwang et al. (2007); Xu et al. (2009); Zhao et al. (2006); Savazzi \& Spagnolini (2008); Michalopoulos \& Karagiannidis (2008); Zou et al. (2009); Vicario et al. (2009). The outage probability for repetition-based AF relaying over Rayleigh channel is provided in Zhao et al. (2007). The outage probability of AF relaying over Rician fading is provided only in few articles Zhu et al. (2008). The asymmetric fading channel, mix of Rayleigh and additive white Gaussian noise, is introduced in Katz \& Shamai (2009). The performance of AF relaying over asymmetric fading, mix of Rician \& Rayleigh, is provided in independent work in Suraweera et al. (2009); Suraweera, Karagiannidis \& Smith (2009). However, in the literature, none of the papers provided any closed form outage probability of AF relaying over asymmetric fading channels.

In this work, we provide a complete study of outage probability of repetition-based and opportunistic relaying over asymmetric fading channels. The closed form of outage probability is derived over i.n.d fading channel at high SNR regime. In this work, we adopted AF relaying networks over two different scenarios, called asymmetric channel I and asymmetric channel II given in Fig. 1. We provide analytical model of each of the asymmetric channel and verified through the Monte-Carlo simulation studies. The obtained results of asymmetric fading channel are compared with symmetric fading channel, i.e., with Rician fading channel and Rayleigh fading channel. When outage performance is compared between two asymmetric channels, asymmetric channel I provides better outage performance than asymmetric channel II and when it is compared between two diversity techniques, opportunistic AF relaying provides better outage performance than the repetition-based AF relaying.

The rest of the article is organized as follows. Section 2 discusses a two-hop AF relaying network and asymmetric channel models. Section 3 derives the outage probability of the repetition-based AF relaying over two different asymmetric fading channel scenarios at high SNR regime. Section 4 derives the outage probability of opportunistic AF relaying over asymmetric channel I and asymmetric channel II. Finally, conclusion is drawn in section 6 .

\section{System model}

\subsection{Signal model of AF relaying}

In this framework, we consider a general 2-hop AF relaying network consisting of $S, M$ relays, $\mathrm{R}_{i}, \mathrm{i}=1,2, \ldots, M$, and $\mathrm{D}$. We assume that $\mathrm{D}$ performs maximal ratio combining at the receiver. The network has $M+1$ time slots for $M$ relay nodes $Z$ hao et al. (2005). In the first time slot, $S$ broadcasts data to $D$ and all $R_{i}$. The received signals at $D$ and $R_{i}$ are given by

$$
\begin{gathered}
y_{s d}=h_{s d} x+\eta_{d} \\
y_{s r_{i}}=\alpha h_{s r_{i}} x+\eta_{r_{i}}
\end{gathered}
$$


where $x$ is the signal transmitted by $S, \eta_{d}$ and $\eta_{r_{i}}$ are the zeromean complex Gaussian random variables at D and $i^{\text {th }}$ relay, respectively. $h_{s d}$ and $h_{s r i}$ are the fading coefficients of S-D and S$\mathrm{R}_{i}$ links, respectively.

The received signal at $\mathrm{D}$ from $\mathrm{R}_{i}$ at the $(i+1)^{\text {th }}$ time slot is

$$
y_{r_{i} d}=h_{r_{i} d} x^{\prime}+\eta_{d}
$$

where $\alpha=1, x^{\prime}$ is the signal transmitted by the relay $\mathrm{R}_{\mathrm{i}}$ for the case of repetition-based relaying and $h_{r i d}$ is the fading coefficient of $\mathrm{R}_{i}$-D link. For the opportunistic AF relaying, $\alpha$ is the amplifying factor and $x^{\prime}=x$. In the $2^{\text {nd }}$ time slot, the best opportunistic relay node forwards the source's signal to D.

\subsection{Channel model of asymmetric fading channel}

For simplicity, we use different notations of the random variables for different fading distributions. For the Rayleigh channel, in general, let $\gamma_{a b}=P_{s}\left|h_{a b}\right|^{2}$ be the instantaneous signal power of a-b link and for the Rician fading channel, the corresponding instantaneous signal power is denoted as $\xi_{a b}$. The transmitted power from source and relay is $P_{s}$. The probability density function (PDF) of $\gamma_{a b}$ and $\xi_{a b}$ are expressed respectively as

$$
\begin{gathered}
f_{\gamma_{a b}}(x)=\frac{1}{\bar{\gamma}_{a b}} e^{-x / \bar{\gamma}_{a b}} \\
f_{\xi_{a b}}(\xi)=\frac{K_{a b}+1}{\bar{\xi}_{a b}} e^{-\xi\left(K_{a b}+1\right) / \bar{\xi}_{a b}-K_{a b}} I_{0}\left(\sqrt{\frac{4 K_{a b}\left(K_{a b}+1\right) \xi}{\bar{\xi}_{a b}}}\right)
\end{gathered}
$$

where $I_{0}($.$) is the 0^{\text {th }}$ order modified Bessel function of first kind, $\bar{\gamma}_{a b}=E\left\{\gamma_{a b}\right\}, \bar{\xi}_{a b}=E\left\{\xi_{a b}\right\}$, and $K_{a b}$ is the Rician factor.

Although there are several possibilities of asymmetric fading channel, in this work, we assume two asymmetric fading channels: namely asymmetric channel I and asymmetric channel II, shown in Fig. 1. For the asymmetric channel I, we assume that S-R link undergos Rayleigh distribution and S-D and R-D links undergo Rician fading distribution. For the asymmetric channel II, S-R link undergos Rician distribution and S-D and R-D links undergo Rayleigh distribution.

\section{Repetition based AF relaying}

The repetition-based AF relaying is introduced in Laneman et al. (2004). Due to the higher degree of freedom of repetition-based relaying and simple implementation for AF relaying, it gain its own importance in cooperative communications. The equivalent instantaneous end-toend signal-to-noise ratio (SNR) for repetition-based AF relaying is given as Zhao et al. (2006)

$$
\gamma=\frac{P_{s}\left|h_{s d}\right|^{2}}{N_{s d}}+\sum_{i=1}^{M} \frac{\frac{P_{s}\left|h_{s r_{i}}\right|^{2}}{N_{s r_{i}}} \frac{P_{s}\left|h_{r_{i} d}\right|^{2}}{N_{r_{i} d}}}{\frac{P_{s}\left|h_{s r_{i}}\right|^{2}}{N_{s r_{i}}}+\frac{P_{s}\left|h_{r_{i} d}\right|^{2}}{N_{r_{i} d}}+1}
$$




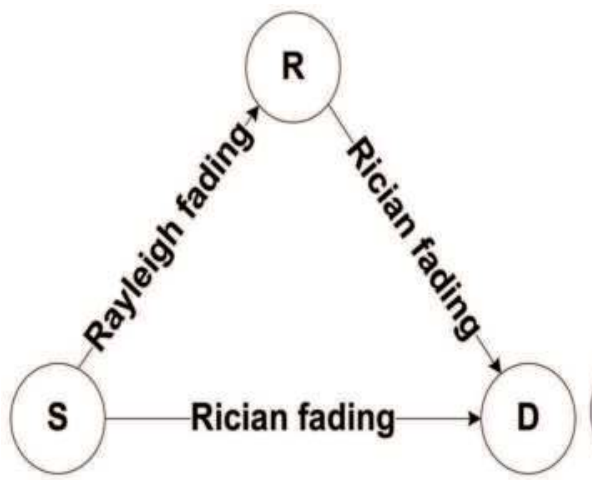

Asymmetric channel I

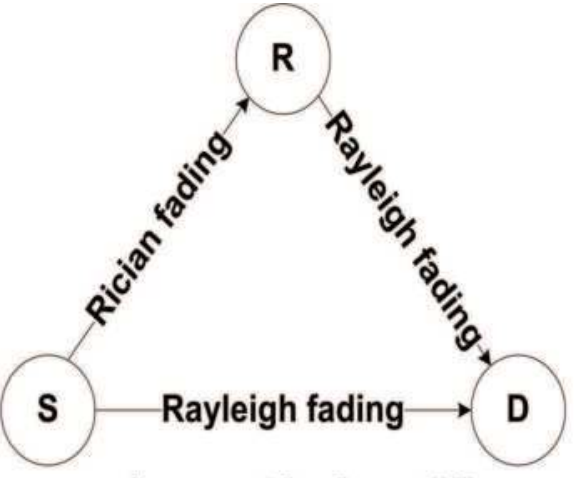

Asymmetric channel II

Fig. 1. Different asymmetric fading channels of a cooperative networks

The upper bound of instantaneous SNR for the above can be written as

$$
\gamma_{\max }=P_{s}\left|h_{s d}\right|^{2} \gamma_{0}+\sum_{i=1}^{M} \min \left(P_{s}\left|h_{s r_{i}}\right|^{2} \gamma_{0}, P_{s}\left|h_{r_{i} d}\right|^{2} \gamma_{0}\right)
$$

In the following section, we provide the lower bound of outage probability of the opportunistic AF relaying for different asymmetric fading channels shown in Fig. 1.

\subsection{Asymmetric channel I:}

For the asymmetric channel I, S-R link experiences Rayleigh distribution and S-D and R-D links experience Rician fading distribution, so (7) can be written as

$$
\gamma_{\max }=\gamma_{0} \gamma_{s d}+\gamma_{0} \xi_{\text {sum }}
$$

where $\gamma_{s d}=P_{a}\left|h_{s d}\right|^{2}$ is the exponential distribution, $\xi_{s u m}=\sum_{i=1}^{M} \xi_{\min , i}, \xi_{\min , i}=\min \left(\xi_{s r_{i}}, \xi_{r_{i} d}\right), \xi_{s r_{i}}$ and $\xi_{r_{i} d}$ are the random variables of noncentral Chi-square distribution. The corresponding outage probability can be defined as

$$
p_{\text {out }}=\operatorname{Pr}\left[\gamma_{u b}<\gamma\right]
$$

where $\gamma_{u b}=\gamma_{\max } / \gamma_{0}, \gamma=\left(2^{(M+1) R}-1\right) / \gamma_{0}$. The outage probability provided in (9) is equivalent to the commutative distribution function (CDF) of $\gamma_{u b}$. The direct evaluation CDF of $\gamma_{u b}$ is complicated, so we use the initial value theorem (IVT) of Laplace transformation (LT). Therefore, this derived analytical results are valid for high SNR regime. To evaluate this, first CDF of $\xi_{\min , i}$ needs to be evaluated. The CDF of the random variable $\xi_{\min , i}$ can be written as

$$
\begin{aligned}
F_{\xi_{\text {min }, i}}(\gamma) & =1-\left(1-\operatorname{Pr}\left[\xi_{s r_{i}}<\gamma\right]\right)\left(1-\operatorname{Pr}\left[\xi_{r_{i} d}<\gamma\right]\right) \\
& =1-Q_{1}\left(\sqrt{2 K_{s r_{i}}}, \sqrt{\frac{2\left(K_{s r_{i}}+1\right) \gamma}{\bar{\xi}_{s r_{i}}}}\right) Q_{1}\left(\sqrt{2 K_{r_{i} d}}, \sqrt{\frac{2\left(K_{r_{i} d}+1\right) \gamma}{\xi_{r_{i} d}}}\right)
\end{aligned}
$$


where $Q_{1}($.$) is the Marcum Q-function of first order. The PDF of \xi_{\text {min, } i}$ is obtained by differentiating the above, which can be expresses as

$$
f_{\xi_{\text {min }, i}}(\gamma)=Q_{1}\left(\sqrt{2 K_{s r_{i}}}, \sqrt{\frac{2\left(K_{s r_{i}}+1\right) \gamma}{\bar{\xi}_{s r_{i}}}}\right) f_{\xi_{r_{i} d}}(\gamma)+Q_{1}\left(\sqrt{2 K_{r_{i} d}}, \sqrt{\frac{2\left(K_{r_{i} d}+1\right) \gamma}{\bar{\xi}_{r_{i} d}}}\right) f_{\xi_{s r_{i}}}(\gamma)
$$

The LT of the random variable $\gamma_{u b}=\gamma_{s d}+\xi_{\text {sum }}$ is obtained by using IVT at high SNR regime. Since $\gamma \rightarrow 0$ as $\gamma_{0} \rightarrow \infty$, we can write

$$
\lim _{s \rightarrow \infty} s \mathcal{L}\left(f_{\gamma_{s d}}(\gamma)\right)=\lim _{\gamma \rightarrow \infty} f_{\gamma_{s d}}(\gamma)
$$

This implies

$$
\mathcal{L}\left(f_{\gamma_{s d}}(\gamma)\right)=\frac{1}{S} f_{\gamma_{s d}}(0)
$$

Similarly the LT of the PDF of random variable $\xi_{\text {sum }}$ can be expressed as

$$
\mathcal{L}\left(f_{\xi_{\text {sum }}}(\gamma)\right)=\frac{1}{s^{M}} \prod_{i=1}^{M} f_{\xi_{\text {min }, i}}(0)
$$

Now by using the multiplication properties of LT, the LT of the PDF of random variable $\gamma_{u b}$ for i.n.d fading channel can be written as

$$
\mathcal{L}\left(f_{\gamma_{s d}}(\gamma)\right)=\frac{1}{s^{M+1}} f_{\gamma_{s d}}(0) \prod_{i=1}^{M} f_{\xi_{\text {min }, i}}(0)
$$

The PDF of random variable $\gamma_{u b}$ is obtained by applying inverse LT (ILT) on the above

$$
f_{\gamma_{u b}}(\gamma)=\frac{1}{M !} \gamma^{M} f_{\gamma_{s d}}(0) \prod_{i=1}^{M} f_{\xi_{\text {min, }}}(0)
$$

By integrating the above and substituting the value of $f_{\gamma_{s d}}(0)$ and $f_{\xi_{\text {min, }}}(0)$, the outage probability can be expressed as

$$
p_{\text {out }}=\frac{1}{(M+1) ! \bar{\gamma}_{s d}} \prod_{i=1}^{M}\left(\frac{\left(K_{s r_{i}}+1\right)}{\bar{\xi}_{s r_{i}} e^{K_{s r_{i}}}}+\frac{\left(K_{r_{i} d}+1\right)}{\bar{\xi}_{r_{i} d} e^{K_{r_{i} d}}}\right) \gamma^{M+1}
$$

\subsection{Asymmetric channel II:}

For asymmetric channel II, signal in S-D and R-D links experience Rayleigh distribution and signal in S-R link experiences Rician distribution. For this scenario, we use $\xi_{s d}=P_{s}\left|h_{s d}\right|^{2}$, $\xi_{s r_{i}}=P_{s}\left|h_{s r_{i}}\right|^{2}$ and $\gamma_{r_{i} d}=P_{s}\left|h_{r_{i} d}\right|^{2}$. The end-to-end instantaneous SNR can be expressed as

$$
\gamma_{u b}=\gamma_{0} \xi_{s d}+\gamma_{0} g_{s u m}
$$


where $g_{\text {sum }}=\sum_{i=1}^{M} g_{\min , i}$ and $g_{\min , i}=\min \left(\xi_{s r_{i}}, \gamma_{r_{i} d}\right)$. Similarly as the previous section, the PDF of $g_{\text {min, }, i}$ is expressed as

$$
f_{g_{\text {min }, i}}(\gamma)=Q_{1}\left(\sqrt{2 K_{s r_{i}}}, \sqrt{\frac{2\left(K_{s r_{i}}+1\right) \gamma}{\bar{\xi}_{s r_{i}}}}\right) f_{\gamma_{r_{i} d}}(\gamma)+f_{\xi_{s r_{i}}}(\gamma)\left(1-F_{\gamma_{r_{i} d}}(\gamma)\right)
$$

where $F_{\gamma_{r_{i} d}}(\gamma)$ is the CDF of the random variable $\gamma_{r_{i} d}$.

Similarly as previous, the PDF of $\gamma_{u b}$ for this asymmetric channel can be derived as

$$
f_{\gamma_{u b}}(\gamma)=\frac{1}{M !} \gamma^{M} f_{\xi_{s d}}(0) \prod_{i=1}^{M} f_{g_{\text {min }, i}}(0)
$$

By integrating (20), the outage probability for the asymmetric channel II can be expressed as

$$
p_{\text {out }}=\frac{1}{(M+1) !} \frac{\left(K_{s d}+1\right)}{\bar{\xi}_{s d} e^{K_{s d}}} \prod_{i=1}^{M}\left(\frac{\left(K_{s r_{i}}+1\right)}{\bar{\xi}_{s r_{i}} e^{K_{s r_{i}}}}+\frac{1}{\bar{\gamma}_{r_{i} d}}\right) \gamma^{M+1}
$$

\section{Opportunistic AF relaying}

In this section, we analyze the outage probability of opportunistic AF relaying over the same asymmetric fading scenario. For the relay selection, we use maximum SNR approach provided in Bletsas et al. (2007). The equivalent instantaneous end-to-end SNR for opportunistic AF relaying is given as Zhao et al. (2006)

$$
\gamma=\frac{P_{s}\left|h_{s d}\right|^{2}}{N_{s d}}+\max _{i=\{1,2, \ldots, M\}} \frac{\frac{P_{s}\left|h_{s r_{i}}\right|^{2}}{N_{s r_{i}}} \frac{P_{s}\left|h_{r_{i} d}\right|^{2}}{N_{r_{i} d}}}{\frac{P_{s}\left|h_{s r_{i}}\right|^{2}}{N_{s r_{i}}}+\frac{P_{s}\left|h_{r_{i} d}\right|^{2}}{N_{r_{i} d}}+1}
$$

The upper bound of instantaneous SNR for the above can be written as

$$
\gamma_{\max }=P_{s}\left|h_{s d}\right|^{2} \gamma_{0}+\max _{i=\{1,2, \ldots, M\}} \min \left(P_{s}\left|h_{s r_{i}}\right|^{2} \gamma_{0}, P_{s}\left|h_{r_{i} d}\right|^{2} \gamma_{0}\right)
$$

\subsection{Asymmetric channel I:}

In asymmetric channel I, the outage performance can be expressed as

$$
p_{\text {out }}=\operatorname{Pr}\left[\gamma_{u b}<\gamma\right]
$$

where $\gamma_{u b}=\gamma_{\max } / \gamma_{0}, \gamma=\left(2^{2 R}-1\right) / \gamma_{0}, \xi_{\max }=\max \left(\xi_{\min , 1}, \xi_{\min , 2}, \ldots, \xi_{\min , M}\right)$ and $\xi_{\min , i}=\min \left(\xi_{s r_{i}}, \xi_{r_{i} d}\right)$. The CDF of the random variable $\xi_{\max }$ for i.n.d fading channel can be expressed as

$$
F_{\xi_{\max }}(\gamma)=\prod_{i=1}^{M} F_{\xi_{\text {min, }}}(\gamma)
$$


and the corresponding PDF of $\xi_{\max }$ is obtained as

$$
f_{\xi_{\max }}(\gamma)=\sum_{i=1}^{M} f_{\xi_{\min , i}}(\gamma) \prod_{\substack{j=1 \\ j \neq i}}^{M} f_{\xi_{\min , j}}(\gamma)
$$

Since $F_{\xi_{\text {min, }}}(0)=0$, the $(M-1)^{\text {th }}$ order derivative of (26) at high SNR, i.e., at $\gamma=0$ for $\gamma_{0} \rightarrow \infty$, can be derived as

$$
\left.\frac{\partial^{M-1}}{\partial \gamma^{M-1}} f_{\xi_{\max }}(\gamma)\right|_{\gamma=0}=M ! \prod_{i=1}^{M} f_{\xi_{\min , i}}(0)
$$

By using LT of $\mathrm{M}^{\text {th }}$ order differentiation, we can write

$$
\mathcal{L}\left(\frac{\partial^{M-1}}{\partial \gamma^{M-1}} f_{\xi_{\max }}(\gamma)\right)=s^{M-1} \mathcal{L}\left(f_{\gamma_{\max }}(\gamma)\right)-s^{M-2} f_{\gamma_{\max }}(0)-\ldots-f_{\gamma_{\max }}^{(M-2)}(0)
$$

Since $f_{\gamma_{\max }}(0)=f_{\gamma_{\max }}^{(1)}(0)=\ldots=f_{\gamma_{\max }}^{(M-2)}(0)=0$, by using the IVT of LT, we can write

$$
\underset{\lim s \rightarrow \infty}{\mathcal{L}}\left(f_{\gamma_{\max }}(\gamma)\right)=\left.\frac{1}{s^{M}} \frac{\partial^{M-1}}{\partial \gamma^{M-1}} f_{\xi_{\max }}(\gamma)\right|_{\gamma=0}
$$

The LT of the PDF of random variable $\gamma_{u b}=\gamma_{s d}+\xi_{\max }$ over i.n.d can be written as

$$
\begin{aligned}
\mathcal{L}\left(f_{\gamma_{u b}}(\gamma)\right) & =\mathcal{L}\left(f_{\gamma_{\text {sum }}}(\gamma)\right) \mathcal{L}\left(f_{\gamma_{\max }}(\gamma)\right) \\
& =\left.\frac{1}{s^{M+1}} f_{\gamma_{s d}}(0) \frac{\partial^{M-1}}{\partial \gamma^{M-1}} f_{\xi_{\max }}(\gamma)\right|_{\gamma=0} \\
& =\frac{M !}{s^{M+1}} f_{\gamma_{s d}}(0) \prod_{i=1}^{M} f_{\xi_{\min , i}}(0)
\end{aligned}
$$

with respect to $s$, the PDF of $\gamma_{u b}$ is obtained by applying the ILT on the above as

$$
f_{\gamma_{u b}}(\gamma)=\gamma^{M} f_{\gamma_{s d}}(0) \prod_{i=1}^{M} f_{\xi_{\text {min,i }}}(0)
$$

The corresponding outage probability or CDF of $\gamma_{u b}$ is obtained by integrating the above as.

$$
p_{\text {out }}=\frac{1}{(M+1) \bar{\gamma}_{s d}} \prod_{i=1}^{M}\left(\frac{K_{s r_{i}}+1}{\bar{\xi}_{s r_{i}} e^{K_{r_{i}}}}+\frac{K_{r_{i} d}+1}{\bar{\xi}_{r_{i} d} e^{K_{r_{i} d}}}\right) \gamma^{M+1}
$$

\subsection{Asymmetric channel II:}

Similarly, in asymmetric channel II, the outage performance can be expressed as

$$
p_{\text {out }}=\operatorname{Pr}\left[\gamma_{u b}<\gamma\right]
$$


where $\gamma_{u b}=\xi_{s d}+g_{\max }, g_{\max }=\max \left(g_{\min , 1}, g_{\min , 2}, \ldots, g_{\min , M}\right)$ and $g_{\min , i}=\min \left(\gamma_{0} \xi_{s r_{i}}, \gamma_{0} \gamma_{r_{i} d}\right)$.

As the previously, the LT of the random variable of $\gamma_{u b}$ over i.n.d fading channel can be written as

$$
\mathcal{L}\left(f_{\gamma_{u b}}(\gamma)\right)=\frac{M !}{s^{M+1}} f_{\gamma_{s d}}(0) \prod_{i=1}^{M} f_{g_{\text {min }, i}}(0)
$$

The PDF of $\gamma_{u b}$ is obtained by applying the ILT on the above as

$$
f_{\gamma_{u b}}(\gamma)=\gamma^{M} f_{\gamma_{s d}}(0) \prod_{i=1}^{M} f_{g_{\text {min }, i}}(0)
$$

The corresponding outage probability or CDF of $\gamma_{u b}$ is obtained by integrating the above as

$$
p_{\text {out }}=\frac{1}{(M+1) \bar{\gamma}_{s d}} \prod_{i=1}^{M}\left(\frac{K_{s r_{i}}+1}{\bar{\xi}_{s r_{i}} e^{K_{s r_{i}}}}+\frac{1}{\bar{\gamma}_{r_{i} d}}\right) \gamma^{M+1}
$$

\section{Numericale}

In this section, analytical and Monte-Carlo simulation results are presented. Since the channel are i.n.d, we set different means for different $S-\mathrm{R}_{i} / \mathrm{R}_{i}-\mathrm{D}$ links. In the Rician fading channel, the Rician factor $K_{a b}$ is uniformly distributed in [2,3] and the mean $\bar{\gamma}_{a b}$ of NLOS components are uniformly distributed in $[0,1]$. The LOS components are derived for a given value of $K_{a b}$ and $\bar{\gamma}_{a b}$. The number of relay nodes is set to 2, 4, 5 and 6 .

Fig. 2, Fig. 3 and Fig. 4 show the lower bound of outage probability of repetition-based AF relaying over asymmetric channel I, asymmetric channel II and a comparison among the different fading channels. Since the analytical outage probability are derived based on high SNR assumption, analytical results converge with Monte-Carlo simulation results at high SNR value. From Fig. 2 and Fig. 3, it is clear that the diversity of repetition-based AF relaying increases with the number of relay nodes.

From Fig. 4, it is clear that the outage performance over Rician fading channel outperforms all other fading scenarios due to the presence of LOS signal. On the other hand, due to the absence of LOS signals, Rayleigh fading channel has poorer outage performance than all other fading scenarios. When the outage performance is compared between two different asymmetric channels, asymmetric channel I provides better outage performance than the asymmetric channel II. It is because of S-R link experience LOS signal in asymmetric channel I, so, there is less chance to amplify the noise by relay nodes. However, for the asymmetric channel II, S-R is a NLOS situation, there is more chance to amplify the noise by relay nodes and send it to the destination.

Fig. 5, Fig. 6 and Fig. 7 show the lower bound of outage probability of opportunistic AF relaying over asymmetric channel I, asymmetric channel II and a comparison among the different fading channels. As similar as repetition-based relaying, analytical outage performance converges with Monte-Carlo simulation results at high SNR values. In opportunistic relaying, the performance as well as the diversity increase with the number of 
relay nodes. When the outage performance is compared among the fading channel, opportunistic relaying shows the same characteristic as repetition based relaying. Without providing any extra simulation, it is easily concluded that opportunistic AF relaying provides better outage performance than the repetition-based AF relaying.

\section{Conclusions}

This work investigates the outage performance of repetition-based and opportunistic AF relaying over two different asymmetric fading channel. The lower bound of outage probability is derived for high SNR regime and validated through the Monte-Carlo simulation studies. It is observed that asymmetric channel I has better outage performance than that of asymmetric channel II for both the repetition-based and opportunistic AF relaying, and opportunistic AF relaying provides better outage performance than the repetition-based AF relaying.

\section{Acknowledgments}

The authors would like to thank the European IST-FP7 WHERE project for support of this work.

\section{References}

Bletsas, A., Shin, H. and Win, M. Z. (2007). Cooperative communication with outage optimal opportunistic relaying, IEEE Transactions on Wireless Communications 6: 3450-3459.

Hwang, K.-S., Ko, Y.-C. and Alouini, M.-S. (2007). Outage probability of cooperative diversity systems with opportunistic relaying based on decode-and-forwards, IEEE Transactions on Wireless Communications 7: 5100-5106.

Katz, M. and Shamai, S. (2009). Relaying protocols for two colocated users, IEEE Transactions on Information Theory 52: 2329 - 2344.

Krikidis, I. and Thompson, J. (2008). Amplify-and-Forword with partial realy selection, IEEE Communications Letters 12: 235-237.

Laneman, J. N., Tse, D. N. C. and Wornell, G. W. (2004). Cooperative diversity in wireless networks: Efficient protocols and outage behavior, IEEE Transactions of Information Theory 50: 3062-3080.

Liu, K. J. R., Sadek, A. K., Su, W. and Kwasinski, A. (2009). Cooperative Communications and Networking, Canbridge.

Michalopoulos, D. and Karagiannidis, G. (2008). Performance analysis of single relay selection in Rayleigh fading, IEEE Transactions on Wireless Communications 7(10): 3718-3724.

Nosratinia, A., Hunter, T. E. and Hedayat, A. (2004). Cooperative communication in wireless networks, IEEE Communications Magazine 42: 74-80.

Paulraj, A., Gore, D., Nabar, R. and Bolcskei, H. (2004). An overview of mimo communications - a key to gigabit wireless, Proceedings of the IEEE 92(2): 198-218.

Savazzi, S. and Spagnolini, U. (2008). Cooperative fading regions for decode and forward relaying, IEEE Transactions on Information Theory 54(11): 4908-4924. 
Suraweera, H., Karagiannidis, G. and Smith, P. (2009). Performance analysis of the dualhop asymmetric fading channel, IEEE Transactions on Wireless Communications Letters 8: 2783-2788.

Suraweera, H., Louie, R., Li, Y., Karagiannidis, G. and Vucetic, B. (2009). Two hop amplifyand-forward transmission in mixed Rayleigh and Rician fading channels, IEEE Communications Letters 13(4): 227-229.

Vicario, J., Bel, A., Lopez-Salcedo, J. and Seco, G. (2009). Opportunistic relay selection with outdated csi: outage probability and diversity analysis, IEEE Transactions on Wireless Communications 8(6): 2872-2876.

Xu, F., Lau, F. C. M., Zhou, Q. F. and You, D. W. (2009). Outage peformance of cooperative communication systems using opportunistic relaying and selection combining receiver, IEEE Singal Processing Letters 16: 113-116.

Zhao, Y., Adve, R. and Lim, T. (2007). Improving amplify-and-forward relay networks: optimal power allocation versus selection, IEEE Transactions on Wireless Communications 6(8): 3114-3123.

Zhao, Y., Adve, R. and Lim, T. J. (2005). Outage probability at arbitrary SNR with cooperative diversity, IEEE Communications Letters 9: 700-703.

Zhao, Y., Adve, R. and Lim, T. J. (2006). Symbol error rate of selection Amplify-and-Forward relay systems, IEEE Communications Letters 10: 757-759.

Zhu, Y., Xin, Y. and Kam, P.-Y. (2008). Outage probability of Rician fading relay channels, IEEE Transactions on Vehicular Technology 57(4): 2648-2652.

Zou, Y., Zheng, B. and Zhu, J. (2009). Outage analysis of opportunistic cooperation over Rayleigh fading channels, IEEE Transactions on Wireless Communications 8(6): 30773085 . 


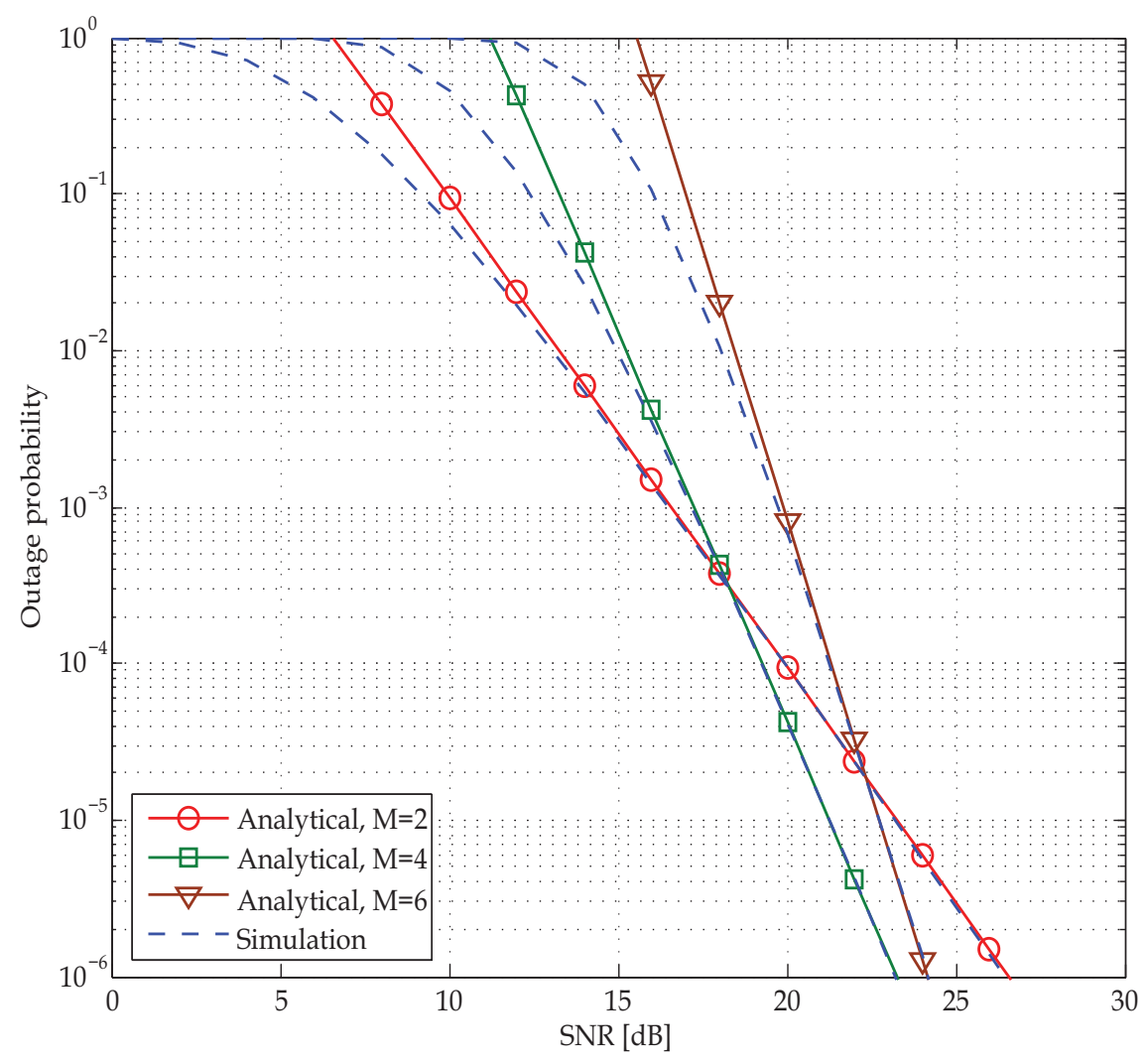

Fig. 2. The outage probability of repetition-based AF relaying over asymmetric channel I. The number of relay node is selected $M=2, M=4$ and $M=6$. 


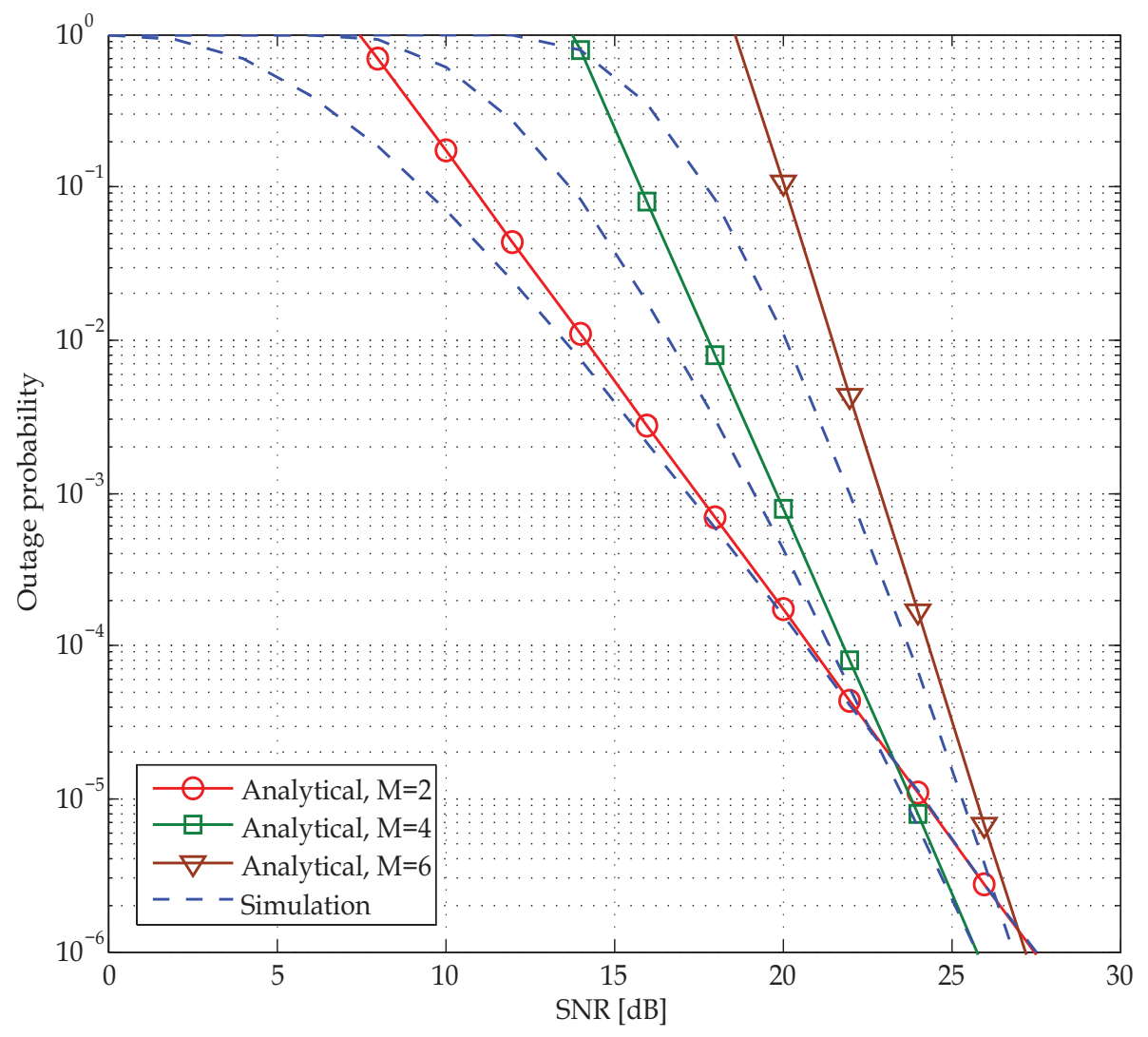

Fig. 3. The outage probability of repetition-based AF relaying over asymmetric channel II. The number of relay node is selected $M=2, M=4$ and $M=6$. 


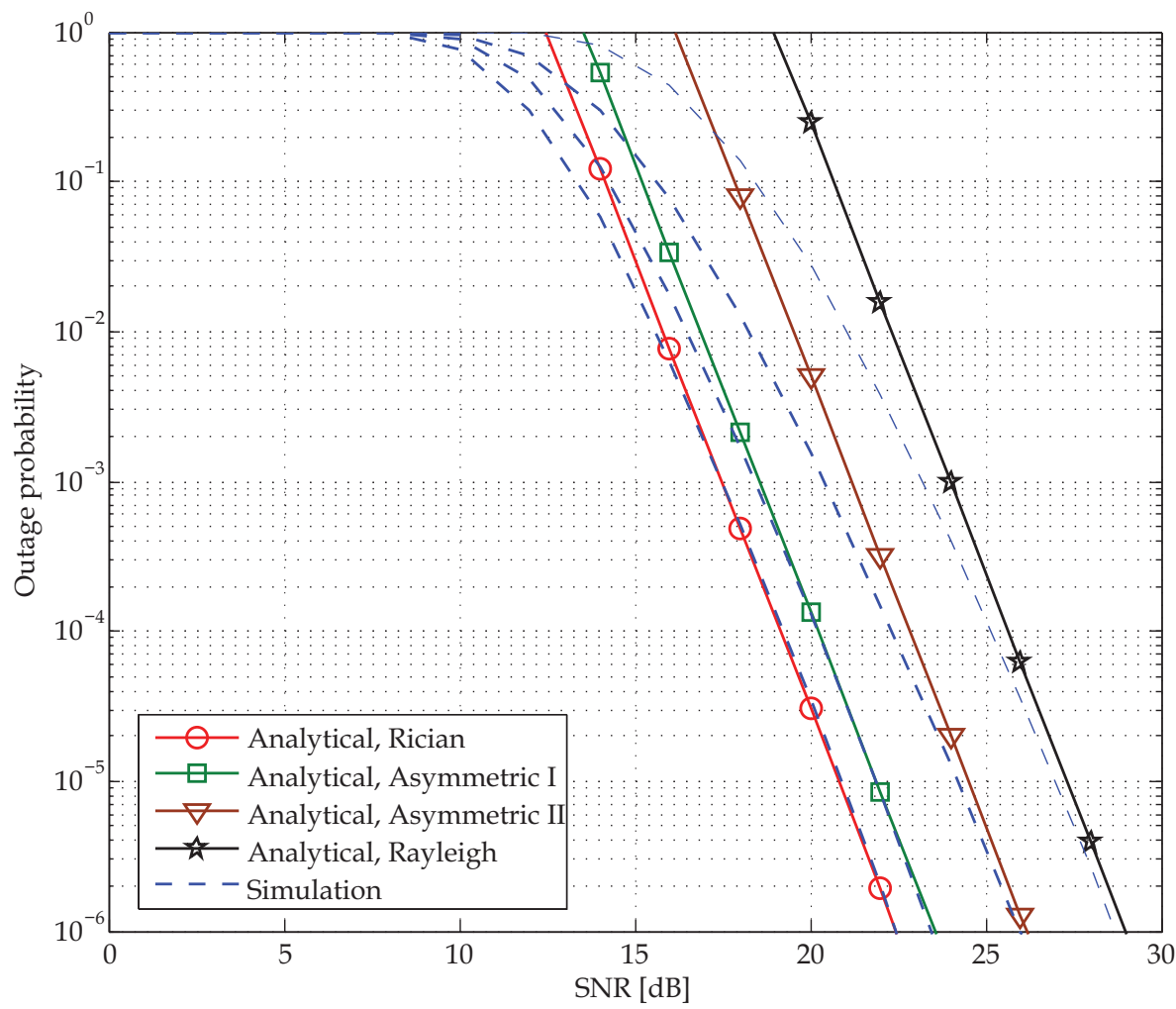

Fig. 4. The comparison of outage probability of repetition-based relaying over different fading channel such as Rician fading, Rayleigh fading, asymmetric channel I and asymmetric channel II. The number of relay nodes is of $M=5$. 


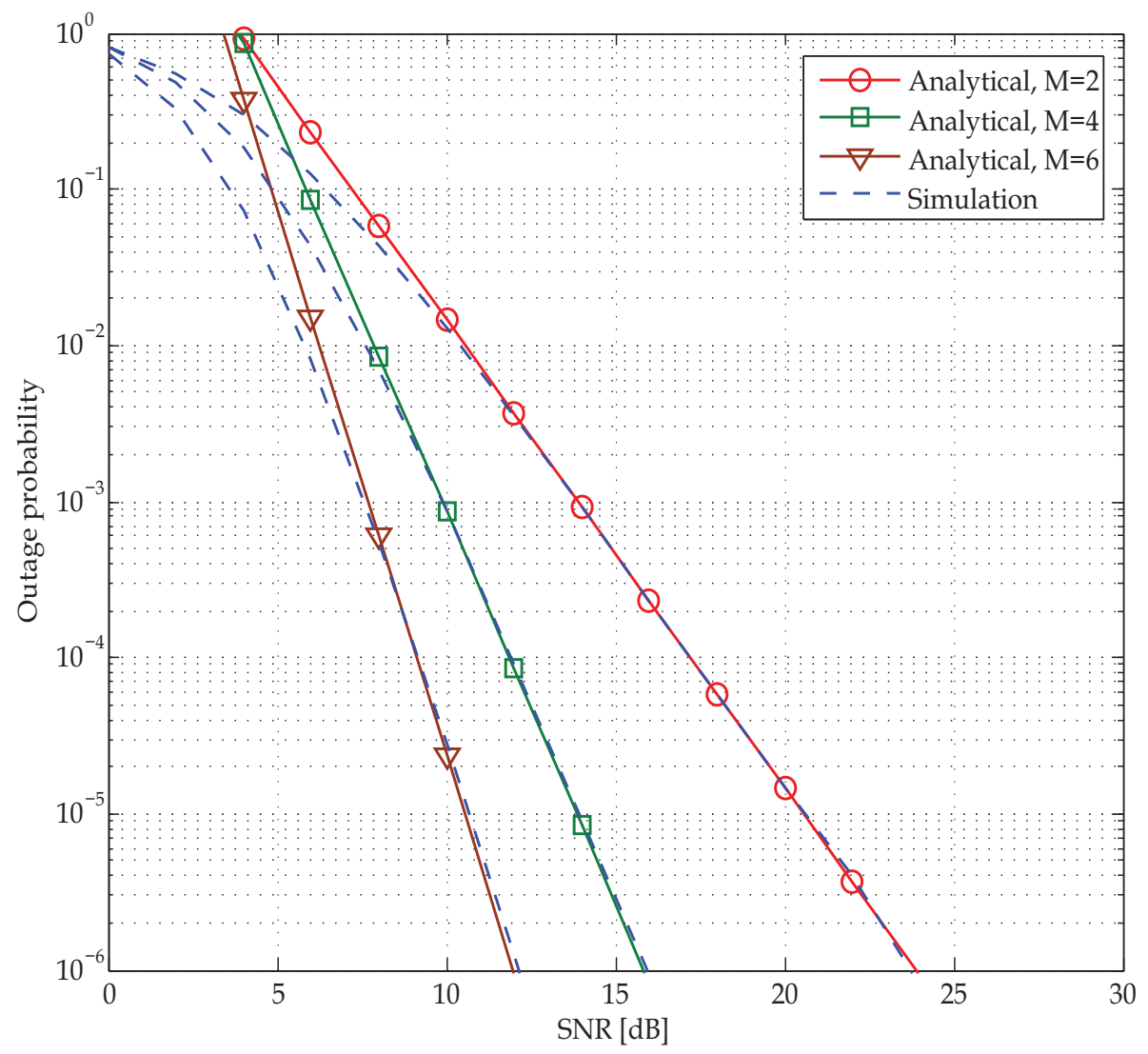

Fig. 5. The outage probability of opportunistic AF relaying over asymmetric channel I. The number of relay node is selected $M=2, M=4$ and $M=6$. 


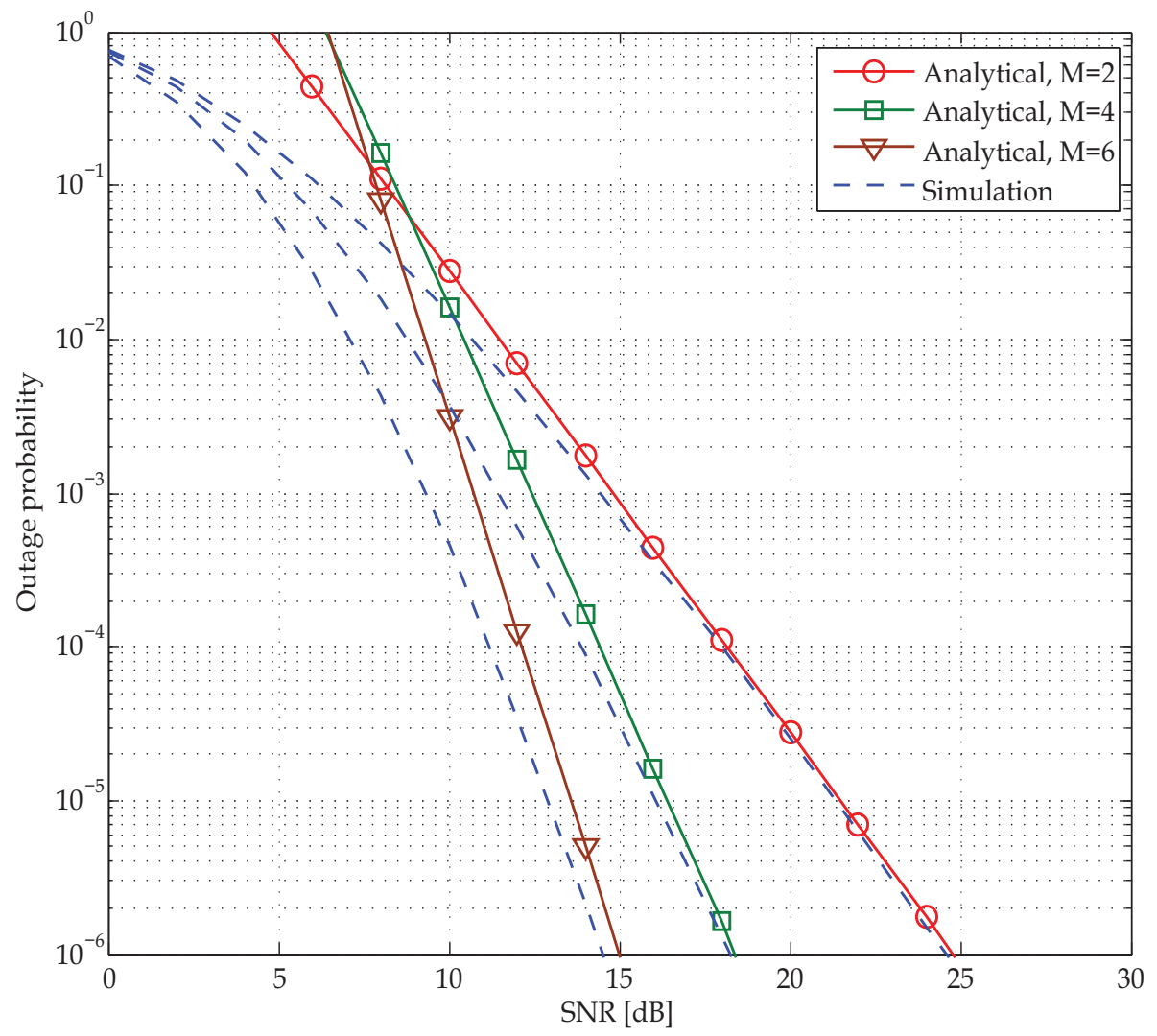

Fig. 6. The outage probability of opportunistic AF relaying over asymmetric channel II. The number of relay node is selected $M=2, M=4$ and $M=6$. 


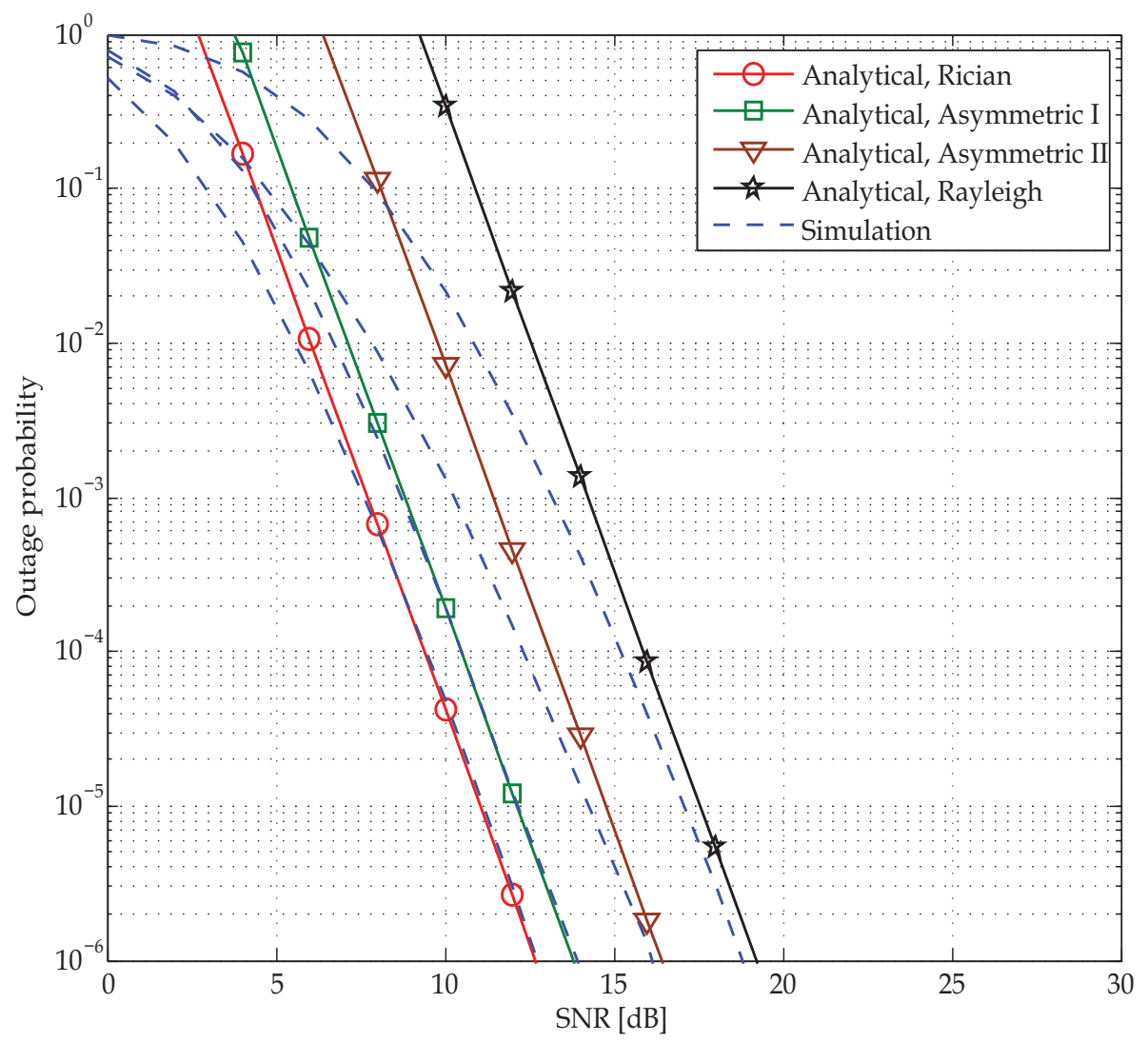

Fig. 7. The comparison of outage probability of opportunistic relaying over different fading channel such as Rician fading, Rayleigh fading, asymmetric channel I and asymmetric channel II. The number of relay nodes is of $M=5$. 


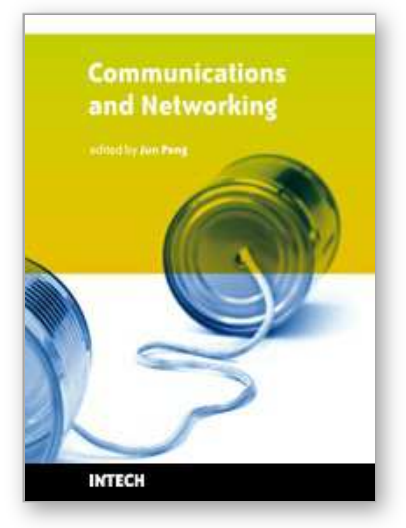

\section{Communications and Networking \\ Edited by Jun Peng}

ISBN 978-953-307-114-5

Hard cover, 434 pages

Publisher Sciyo

Published online 28, September, 2010

Published in print edition September, 2010

This book "Communications and Networking" focuses on the issues at the lowest two layers of communications and networking and provides recent research results on some of these issues. In particular, it first introduces recent research results on many important issues at the physical layer and data link layer of communications and networking and then briefly shows some results on some other important topics such as security and the application of wireless networks. In summary, this book covers a wide range of interesting topics of communications and networking. The introductions, data, and references in this book will help the readers know more abut this topic and help them explore this exciting and fast-evolving field.

\section{How to reference}

In order to correctly reference this scholarly work, feel free to copy and paste the following:

Youssef Nasser, Sudhan Majhi and Jean-Francois Helard (2010). Outage Probability Analysis of Cooperative Communications over Asymmetric Fading Channel, Communications and Networking, Jun Peng (Ed.), ISBN: 978-953-307-114-5, InTech, Available from: http://www.intechopen.com/books/communications-andnetworking/outage-probability-analysis-of-cooperative-communications-over-asymmetric-fading-channel

\section{INTECH}

open science | open minds

\section{InTech Europe}

University Campus STeP Ri Slavka Krautzeka 83/A 51000 Rijeka, Croatia Phone: +385 (51) 770447 Fax: +385 (51) 686166 www.intechopen.com

\section{InTech China}

Unit 405, Office Block, Hotel Equatorial Shanghai No.65, Yan An Road (West), Shanghai, 200040, China 中国上海市延安西路65号上海国际贵都大饭店办公楼 405 单元 Phone: +86-21-62489820

Fax: +86-21-62489821 
(C) 2010 The Author(s). Licensee IntechOpen. This chapter is distributed under the terms of the Creative Commons Attribution-NonCommercialShareAlike-3.0 License, which permits use, distribution and reproduction for non-commercial purposes, provided the original is properly cited and derivative works building on this content are distributed under the same license. 